\title{
Barriers to Implementation of Evidence Based Practice in Zahedan Teaching Hospitals, Iran, 2014
}

\author{
Mohammad Khammarnia, ${ }^{1}$ Mahsa Haj Mohammadi, ${ }^{1}$ Zahra Amani, \\ Shahab Rezaeian, ${ }^{1}$ and Fatemeh Setoodehzadeh ${ }^{1,2}$ \\ ${ }^{1}$ Health Promotion Research Center, Zahedan University of Medical Sciences, P.O. Box 98135, Zahedan, Iran
${ }^{2}$ Department of Health Management and Economics, School of Public Health, Tehran University of Medical Sciences,
P.O. Box 14155-6446, Tehran, Iran
}

Correspondence should be addressed to Shahab Rezaeian; shahab.rezayan@gmail.com

Received 28 November 2014; Revised 27 February 2015; Accepted 9 March 2015

Academic Editor: Maria Helena Palucci Marziale

Copyright (c) 2015 Mohammad Khammarnia et al. This is an open access article distributed under the Creative Commons Attribution License, which permits unrestricted use, distribution, and reproduction in any medium, provided the original work is properly cited.

\begin{abstract}
This study aimed to determine the barriers to implementation of EBP among nurses. This cross-sectional study was conducted in Zahedan City, South East of Iran, in 2014. The questionnaire of barriers to implementation of EBP consists of 27 statements which was distributed among 280 nurses. More than half of the participants agreed that $56 \%$ and $57 \%$ of barriers to implementation of evidence based practice are related to organizational and individual aspects, respectively. Participants identified barriers at organizational level included the lack of human resources (78.3\%), lack of internet access at work (72.2\%), and heavy workload (70.0\%). Barrier at individual level included lack of time to read literature (83.7\%), lack of ability to work with computer (68.8\%), and insufficient proficiency in English language (62.0\%). Age, educational level, job experience, and employment status were associated with organizational barriers to implementation of EBP. At the individual level only education was associated with barriers to implementation of EBP. Barriers to implementation of EBP occur at both individual and organizational levels. The indicator of quality in nursing practice is EBP. Hence, familiarity with EBP is recommended for Iranian nurses. In addition, knowledge of barriers will help health care system and policy makers to provide a culture of EBP.
\end{abstract}

\section{Introduction}

Delivery of high-quality and consistent services is a big challenge in the health care system nowadays [1]. Evidence based practice (EBP), a problem-solving approach to patient care based on the best available and valid evidence, leads to enhanced quality of care [2], reduced costs [3], and the individual and professional development of nurses and other health workers [4]. Moreover, EBP based on international standards enhances the quality of clinical practice [5]. EBP has been promoted as a way for clients to receive the best level of care [6].

Nurses are the largest group of health care providers and have a key role in ensuring the promotion of health care [7] and delivering better services [4]. EBP is important to the professional development, responsibility, and capabilities of nurses [5], and it has become an important subject in nursing and has integrated into daily practice [8]. In addition, nurses who practice based on the scientific evidence have been able to make better decisions in services delivery [9] Though nurses generally report positive attitudes and beliefs towards EBP [9-11], previous studies show that nurses were not familiar enough with its principals and they use EBP to a limited extent $[9,12,13]$.

Several studies have found that both human and organizational factors are associated with barriers to the use of EBP including lack of time to read literature, heavy workload, lack of staff experienced in EBP, and lack of resources [11, 14, 15]. A current systematic review showed that there are many barriers to the implementation and use of EBP and concluded that identifying barriers is the first step to removing them [16]. 
Little research has been conducted on EBP beliefs and its use among Iranian nurses. Moreover, the Iranian nursing care system does not provide the incentive for nurses to engage in research [5] and most nurses were not familiar with the concept of EBP [17].

Therefore, the aim of this study was to determine barriers to implementation of evidence based practice in Zahedan teaching hospitals. The results of the study can help managers and policy makers in planning for better use of EBP in nurses and other staff in hospitals.

\section{Methods}

2.1. Study Design. This analytical cross-sectional study was conducted in Zahedan City, South East of Iran, in 2014.

2.2. Setting and Samples. There are six teaching hospitals in the study region. The sample was all nurses who were at work during data collection in the hospitals. The participants' verbal informed consent was obtained and nurses participated voluntarily in the study.

The stratified random sampling method was used as follows. Zahedan City was divided into six hospitals (stratum). The nurses' population in each hospital was considered as study population. Then, based on nurses personnel list (sampling frame) a proportional random sample from each hospital was taken by the stratum's size. Considering the proportion of barriers to implementation of EBP to be $78.6 \%$ [18], the sample size of 270 at $95 \%$ significant levels and error level of 0.05 was calculated and ten additional participants were added to the sample size and the overall sample size reached 280 subjects. Exclusion criteria were nurses who had not been at work during data gathering.

2.3. Measurements. We used the questionnaire of barriers to implementation of EBP that has been tested for reliability and validity in a previous study [18]. In this study, Cronbach's coefficient alpha of 0.81 indicated sufficient reliability of all statements in the questionnaire. Demographic information was collected such as age, sex, education, job experience, and employment status.

The questionnaire of barriers to implementation of EBP consists of 27 statements with two aspects (organizational aspect: 18 statements, and individual aspect: 9 statements). In both organizational and individual aspects respondents were asked to score the level to which they agree (scored as 1), have no opinion (scored as 2), or disagree (scored as 3 ).

2.4. Data Analysis. Descriptive statistics (frequency table, mean and standard deviation) were conducted to describe the background factors and barriers to implementation of EBP. The Chi-square test was also used for data analysis at 0.05 significance levels using the statistical software STATA 11 (StataCorp, College Station, TX, USA).

\section{Results}

The questionnaires were distributed to 280 nurses, with 263 nurses returning the questionnaires (response rate $=$
TABLE 1: Distribution of participant demographics in Zahedan teaching hospitals.

\begin{tabular}{|c|c|c|}
\hline Variables & Number & $\%$ \\
\hline \multicolumn{3}{|l|}{ Age group } \\
\hline$<25 \mathrm{yr}$ & 123 & 46.8 \\
\hline$+26 \mathrm{yr}$ & 140 & 53.2 \\
\hline Mean (SD) & \multicolumn{2}{|c|}{$28.4(5.4)$} \\
\hline \multicolumn{3}{|l|}{ Gender } \\
\hline Male & 78 & 29.7 \\
\hline Female & 185 & 70.3 \\
\hline \multicolumn{3}{|l|}{ Education } \\
\hline Bachelor's degree & 249 & 94.7 \\
\hline Master's degree & 14 & 5.3 \\
\hline \multicolumn{3}{|l|}{ Job experience (years) } \\
\hline$<5$ & 159 & 60.5 \\
\hline $6-10$ & 62 & 23.6 \\
\hline $11-15$ & 23 & 8.7 \\
\hline$>16$ & 19 & 7.2 \\
\hline \multicolumn{3}{|l|}{ Employment status } \\
\hline Temporary & 185 & 70.3 \\
\hline Permanent & 29 & 11.1 \\
\hline Fixed term & 49 & 18.6 \\
\hline \multicolumn{3}{|c|}{$\begin{array}{l}\text { Barriers to implementation EBP associated } \\
\text { with organizational aspects }\end{array}$} \\
\hline Agree & 148 & 56.0 \\
\hline No comment & 70 & 27.0 \\
\hline Disagree & 45 & 17.0 \\
\hline \multicolumn{3}{|c|}{$\begin{array}{l}\text { Barriers to implementation EBP associated } \\
\text { with individual aspects }\end{array}$} \\
\hline Agree & 150 & 57.0 \\
\hline No comment & 58 & 22.0 \\
\hline Disagree & 55 & 21.0 \\
\hline
\end{tabular}

EBP: evidence based practice.

93.9\%). Table 1 presents the distribution of demographic characteristics of the participants.

The sample included $70.3 \%$ women. The average age was 28.4 years $(\mathrm{SD}=5.4)$ with a range of $21-50$ years. Most of the participants were employed with education level of Bachelor's degree (94.7\%). Most of them (60.5\%) had job experience less than 5 years.

More than half of the participants agreed that $56 \%$ and $57 \%$ of barriers to implementation of evidence based practice are related to organizational and individual aspects, respectively.

The list of five common barriers to implementation of evidence based practice (EBP) by organizational and individual aspects is presented in Table 2. The lack of human resources (shortage of nurse) (78.3\%), lack of internet access at work $(72.2 \%)$, and heavy workload $(70.0 \%)$ are the most important organizational barriers. The most important individual barriers to implementation of EBP are lack of time to read literature $(83.7 \%)$, lack of ability to work with computer 
TABLE 2: The list of five common barriers to implementation of evidence based practice (EBP) by organizational and individual aspects.

\begin{tabular}{|c|c|c|c|}
\hline & Agree & No comment & Disagree \\
\hline \multicolumn{4}{|l|}{ Organizational aspects } \\
\hline Lack of human sources & $206(78.3)$ & $44(16.7)$ & $13(5.0)$ \\
\hline Heavy workload & $190(72.2)$ & $39(14.8)$ & $34(13.0)$ \\
\hline Lack of access to a rich library with nursing journals & $184(70.0)$ & $57(21.7)$ & $22(8.3)$ \\
\hline Lack of internet access at work & $167(63.5)$ & $57(21.7)$ & $39(14.8)$ \\
\hline No cooperation by physicians & $163(62.0)$ & $63(24.0)$ & $37(14.0)$ \\
\hline \multicolumn{4}{|l|}{ Individuals aspects } \\
\hline Lack of time to read literature & $220(83.7)$ & $28(10.7)$ & $15(5.6)$ \\
\hline Insufficient proficiency in English language & $181(68.8)$ & $32(12.2)$ & $50(19.0)$ \\
\hline Lack of ability to work with computer & $163(62.0)$ & $43(16.3)$ & $57(21.7)$ \\
\hline Lack of autonomy to change practice & $145(55.2)$ & $59(22.4)$ & $59(22.4)$ \\
\hline Lack of knowledge & $143(54.4)$ & 47 (17.9) & $73(27.7)$ \\
\hline
\end{tabular}

Figures are number (\%).

TABLE 3: Associations between background variables and implementation of evidence based practice by organizational and individual aspects using Chi-square test.

\begin{tabular}{|c|c|c|c|c|c|c|}
\hline & \multicolumn{3}{|c|}{ Organizational aspects } & \multicolumn{3}{|c|}{ Individual aspects } \\
\hline & Agree & Disagree & $P$ value & Agree & Disagree & $P$ value \\
\hline \multicolumn{7}{|l|}{ Age group (years) } \\
\hline$<25$ & $105(85.4)$ & $18(14.6)$ & \multirow{2}{*}{$0.013^{*}$} & $91(74.0)$ & $32(26.0)$ & \multirow{2}{*}{0.334} \\
\hline$>26$ & $102(72.9)$ & $38(27.1)$ & & $96(68.6)$ & $44(31.4)$ & \\
\hline \multicolumn{7}{|l|}{ Gender } \\
\hline Male & $66(84.6)$ & $12(15.4)$ & \multirow{2}{*}{0.129} & $56(71.8)$ & $22(28.2)$ & \multirow{2}{*}{0.872} \\
\hline Female & $141(76.2)$ & $44(23.8)$ & & $131(70.8)$ & $54(29.2)$ & \\
\hline \multicolumn{7}{|l|}{ Education } \\
\hline Bachelor's degree & $199(79.9)$ & $50(20.1)$ & \multirow{2}{*}{$0.043^{*}$} & $181(72.7)$ & $68(27.3)$ & \multirow{2}{*}{$0.017^{*}$} \\
\hline Master's degree & $8(57.1)$ & $6(42.9)$ & & $6(42.9)$ & $8(57.1)$ & \\
\hline \multicolumn{7}{|l|}{ Job experience (years) } \\
\hline$<5$ & $135(84.9)$ & $24(15.1)$ & \multirow{4}{*}{$0.001^{*}$} & $120(75.5)$ & $39(24.5)$ & \multirow{4}{*}{0.138} \\
\hline $6-10$ & $43(69.4)$ & $19(30.6)$ & & $37(59.7)$ & $25(40.3)$ & \\
\hline $11-15$ & $11(47.8)$ & $12(52.8)$ & & $16(69.6)$ & $7(30.4)$ & \\
\hline+16 & $18(94.7)$ & $1(5.3)$ & & $14(71.1)$ & $5(28.9)$ & \\
\hline \multicolumn{7}{|l|}{ Employment status } \\
\hline Contractual & $155(83.8)$ & $30(16.2)$ & \multirow{3}{*}{$0.008^{*}$} & $134(72.4)$ & $51(27.6)$ & \multirow{3}{*}{0.122} \\
\hline Permanent & $19(65.5)$ & $10(34.5)$ & & $16(55.2)$ & $13(44.8)$ & \\
\hline Official & $33(67.4)$ & $16(32.6)$ & & $37(75.5)$ & $12(24.5)$ & \\
\hline
\end{tabular}

Figures are number $(\%) ;{ }^{*} P$ value $<0.05$.

$(68.8 \%)$, and insufficient proficiency in English language (62.0\%).

Table 3 demonstrates the associations between background variables and barriers to implementation of evidence based practice by organizational and individual aspects. There was a statistical and significant correlation between age, educational level, job experience, employment status, and barriers to implementation of evidence based practice experienced by nurses associated with organizational aspects. With regard to barriers to implementation of evidence based practice experienced by nurses associated with individual aspects, only educational level was significant $(P=0.017)$.

\section{Discussion}

The present study has demonstrated the barriers to implementation of EBP among an Iranian nurses population. The results showed that both organizational and individual aspects are barriers to implementation of EBP.

We found that $57 \%$ of barriers to implementation of EBP are related to individual aspects. The three individual barriers most often encountered are lack of time to read literature, lack of ability to work with computer, and insufficient proficiency in English language. This finding is consistent with other studies. The most commonly reported personal barrier is lack 
of time in different parts of world [19-21]. A qualitative study to explore nurses' experiences and perceptions about EBP showed that over half of participants (52.6\%) had not passed any courses on computers [15]. Previous studies [22, 23] revealed that the important factor to find the best evidence to clinical practice questions is having the ability to work with computers. In addition, an Iranian study reported that $21.4 \%$ of barriers to implementation of EBP are related to individual aspects. The authors showed that lack of time is a common barrier to implementation of EBP [18]. Similar finding is shown in the other medical groups in the literature. For example, a study to determine the barriers of EBP among Iranian urologists found that being familiar with evidence is needed and lack of time is the important barrier to implementation of EBP [24]. Our study also found that language barriers were another important barrier; insufficient familiarity with English language was a significant barrier to EBP implementation. This is supported by studies conducted in Taiwan showing that nurses preferred evidence based resources to be available in Chinese [20,25].

Another important finding of this study was that the 56\% of barriers to the implementation of EBP were related to individual aspects. The most frequently reported organizational barriers to implementation of EBP were lack of human resources (shortage of nurse), lack of internet access at work, heavy workload, and lack of access to a rich library with nursing journals. Varaei et al. [15] reported that in the human resource category, shortage of nurses and heavy workload are the most common barriers to implementation of EBP. Another study exploring the relationship between nurses' personal and professional factors and EBP found that only $32 \%$ of nurses have a library rich in nursing journals at their workplace and $42 \%$ had no internet access [13]. Moreover, the results of a study showed that the most important facilitators to the utilization of research in practice are human resources [5]. Accordingly, organizational support can be a target of change in EBP implementation. In this regard, Schoonover [26] concluded that organizational strategies are needed to influence research awareness and utilization. Another study revealed that several problems of clinical nurse performance appraisal system are related to organizational context. Accordingly, changing of the appraisal system is necessary to support the achievement of high quality of patient care [27].

The background variables such as age, educational level, job experience, and employment status were found to be significantly associated with barriers to implementation of EBP. According to the result, participants with job experience less than five and more than 16 years agree more that organizational aspects are associated with barriers to implementation of EBP. In other words, there was a Ushape association between job experience and organizational barriers of EBP implementation. It was identified that nurses with Bachelor's degree agree more that organizational aspects are associated with barriers to implementation of EBP than those with Master's degree. This issue probably is due to small sample size in subgroup of education. Education level has been mentioned in some of the related studies as a main factor to implementation of EBP [13, 28]. Weng et al. [20] also revealed that academic degree and educational training are important factors to implementation of EBP. Since EBP is a critical and important issue in nursing, nurses should increase their knowledge and attitude about EBP and use it for better delivery services. Beside, policy makers must provide a suitable workplace or opportunities for staff to increase their knowledge in hospitals.

In this study older individuals indicated that organizational aspects are important barriers, more than younger individuals. This result probably has some reasons. Older individuals may be more aware of the current trends or know that EBP is something that they should be doing. Another reason is that they are more familiar with hospital system and factors associated with use of EBP. This finding is inconsistent with research by Thompson et al. revealing that demographic variables are not associated with EBP in nurses [29].

4.1. Limitations. There are some limitations that should be noted. Self-report questionnaire was used to obtain the data which can introduce information biases. For example, the construction of questionnaire, lack of time, and the individual interest may be derived from self-report questionnaire. Another important limitation of our study was the limited sample size in subgroup of variables such as education level (94.7\% versus 5.3\%). Despite these limitations, the high response rate $(93.9 \%)$ could be the strength of this study.

\section{Conclusions}

Barriers to implementation of EBP occur at both individual and organizational levels. The indicator of quality in nursing practice is EBP. Hence, familiarity with EBP is recommended for Iranian nurses. In addition, knowledge of barriers will help health care system and policy makers to address these and to provide a culture of EBP.

\section{Conflict of Interests}

The authors declare that there is no conflict of interests for this study.

\section{Acknowledgment}

The authors would like to thank all nurses who volunteered to participate in our study.

\section{References}

[1] S. M. Shortell, T. G. Rundall, and J. Hsu, "Improving patient care by linking evidence-based medicine and evidence-based management," The Journal of the American Medical Association, vol. 298, no. 6, pp. 673-676, 2007.

[2] J. Grimshaw, M. Eccles, R. Thomas et al., “Toward evidencebased quality improvement: evidence (and its limitations) of the effectiveness of guideline dissemination and implementation strategies 1966-1998," Journal of General Internal Medicine, vol. 21, supplement 2, pp. S14-S20, 2006.

[3] J. C. Fortney, J. M. Pyne, and J. F. Burgess Jr., "Population-level cost-effectiveness of implementing evidence-based practices 
into routine care," Health Services Research, vol. 49, no. 6, pp. 1832-1851, 2014.

[4] A. Heydari, S. R. Mazlom, H. Ranjbar, and L. Scurlock-Evans, "A study of Iranian nurses' and midwives' knowledge, attitudes, and implementation of evidence-based practice: the time for change has arrived," Worldviews on Evidence-Based Nursing, vol. 11, no. 5, pp. 325-331, 2014.

[5] N. Mehrdad, M. Salsali, and A. Kazemnejad, "The spectrum of barriers to and facilitators of research utilization in Iranian nursing," Journal of Clinical Nursing, vol. 17, no. 16, pp. 21942202, 2008.

[6] C. Lyons, T. Brown, M. H. Tseng, J. Casey, and R. Mcdonald, "Evidence-based practice and research utilisation: perceived research knowledge, attitudes, practices and barriers among Australian paediatric occupational therapists," Australian Occupational Therapy Journal, vol. 58, no. 3, pp. 178-186, 2011.

[7] M. A. Hagbaghery, M. Salsali, and F. Ahmadi, "A qualitative study of Iranian nurses' understanding and experiences of professional power," Human Resources for Health, vol. 2, no. 1, article 9, 2004.

[8] J. H. Friesen-Storms, A. Moser, S. van der Loo, A. J. Beurskens, and G. J. Bours, "Systematic implementation of evidence-based practice in a clinical nursing setting: a participatory action research project," Journal of Clinical Nursing, vol. 24, no. 1-2, pp. 57-68, 2015.

[9] K. Stokke, N. R. Olsen, B. Espehaug, and M. W. Nortvedt, "Evidence based practice beliefs and implementation among nurses: a cross-sectional study," BMC Nursing, vol. 13, no. 1, article 8, 2014.

[10] H. S. Thorsteinsson, "Icelandic nurses' beliefs, skills, and resources associated with evidence-based practice and related factors: a national survey," Worldviews on Evidence-Based Nursing, vol. 10, no. 2, pp. 116-126, 2013.

[11] C. E. Brown, M. A. Wickline, L. Ecoff, and D. Glaser, "Nursing practice, knowledge, attitudes and perceived barriers to evidence-based practice at an academic medical center," Journal of Advanced Nursing, vol. 65, no. 2, pp. 371-381, 2009.

[12] M. L. Koehn and K. Lehman, "Nurses' perceptions of evidencebased nursing practice," Journal of Advanced Nursing, vol. 62, no. 2, pp. 209-215, 2008.

[13] M. Mashiach Eizenberg, "Implementation of evidence-based nursing practice: nurses' personal and professional factors?" Journal of Advanced Nursing, vol. 67, no. 1, pp. 33-42, 2011.

[14] N. M. Solomons and J. A. Spross, "Evidence-based practice barriers and facilitators from a continuous quality improvement perspective: an integrative review," Journal of Nursing Management, vol. 19, no. 1, pp. 109-120, 2011.

[15] S. Varaei, M. Salsali, and M. A. Cheraghi, "Implementation of evidence-based nursing practice for diabetic patients: an Iranian experience," International Journal of Nursing Practice, vol. 19, supplement 3, pp. 73-80, 2013.

[16] H. Sadeghi-Bazargani, J. S. Tabrizi, and S. Azami-Aghdash, "Barriers to evidence-based medicine: a systematic review," Journal of Evaluation in Clinical Practice, vol. 20, no. 6, pp. 793802, 2014.

[17] M. Adib-Hajbaghery, "Evidence-based practice: Iranian nurses' perceptions," Worldviews on Evidence-Based Nursing, vol. 6, no. 2, pp. 93-101, 2009.

[18] S. Kermanshahi and A. M. Parvinian, "Barriers to implementation of evidence-based care: viewpoints of nursing staff," Iranian Journal of Medical Education, vol. 12, no. 2, pp. 84-92, 2012.
[19] A. M. Hutchinson and L. Johnston, "Beyond the BARRIERS Scale: commonly reported barriers to research use," The Journal of Nursing Administration, vol. 36, no. 4, pp. 189-199, 2006.

[20] Y.-H. Weng, K. N. Kuo, C.-Y. Yang, H.-L. Lo, C. Chen, and Y.-W. Chiu, "Implementation of evidence-based practice across medical, nursing, pharmacological and allied healthcare professionals: a questionnaire survey in nationwide hospital settings," Implementation Science, vol. 8, no. 1, article 112, 2013.

[21] K. Shuval, A. Shachak, S. Linn, M. Brezis, and S. Reis, "Evaluating primary care doctors' evidence-based medicine skills in a busy clinical setting," Journal of Evaluation in Clinical Practice, vol. 13, no. 4, pp. 576-580, 2007.

[22] H. F. Clarke, C. Bradley, S. Whytock, S. Handfield, R. van der Wal, and S. Gundry, "Pressure ulcers: implementation of evidence-based nursing practice," Journal of Advanced Nursing, vol. 49, no. 6, pp. 578-590, 2005.

[23] K. L. Penz and S. L. Bassendowski, "Evidence-based nursing in clinical practice: implications for nurse educators," Journal of Continuing Education in Nursing, vol. 37, no. 6, pp. 251-254, 2006.

[24] S. Hajebrahimi, F. Sadeghi-Ghyassi, N. Olfati, S. Dastgiri, and L. Maghbouli, "Evidence based practice: perspectives of Iranian urologists," Urology Journal, vol. 10, no. 4, pp. 1099-1105, 2013.

[25] Y.-W. Chiu, Y.-H. Weng, H.-L. Lo, Y.-H. Shih, C.-C. Hsu, and K. N. Kuo, "Comparison of accessing online databases between physicians and nurses in Taiwan," Informatics for Health and Social Care, vol. 37, no. 4, pp. 230-241, 2012.

[26] H. Schoonover, "Barriers to research utilization among registered nurses practicing in a community hospital," Journal for Nurses in Staff Development, vol. 25, no. 4, pp. 199-212, 2009.

[27] N. Nikpeyma, Z. Abed-saeedi, E. Azargashb, and H. Alavi-majd, "Problems of clinical nurse performance appraisal system: a qualitative study," Asian Nursing Research, vol. 8, no. 1, pp. 15-22, 2014.

[28] M. Milner, C. A. Estabrooks, and F. Myrick, "Research utilization and clinical nurse educators: a systematic review," Journal of Evaluation in Clinical Practice, vol. 12, no. 6, pp. 639-655, 2006.

[29] C. Thompson, D. McCaughan, N. Cullum, T. Sheldon, and P. Raynor, "Barriers to evidence-based practice in primary care nursing-why viewing decision-making as context is helpful," Journal of Advanced Nursing, vol. 52, no. 4, pp. 432-444, 2005. 


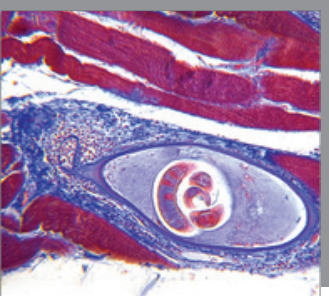

Gastroenterology

Research and Practice
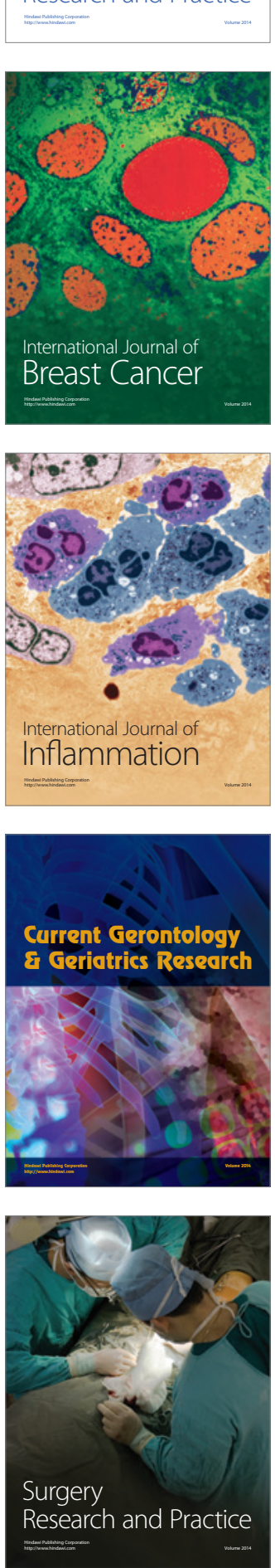

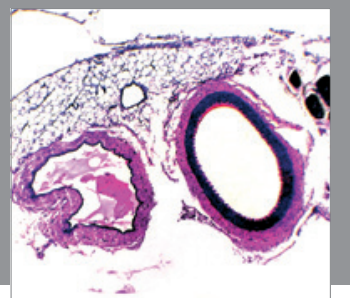

International Journal of Hypertension
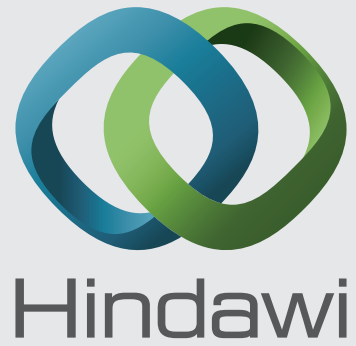

Submit your manuscripts at http://www.hindawi.com
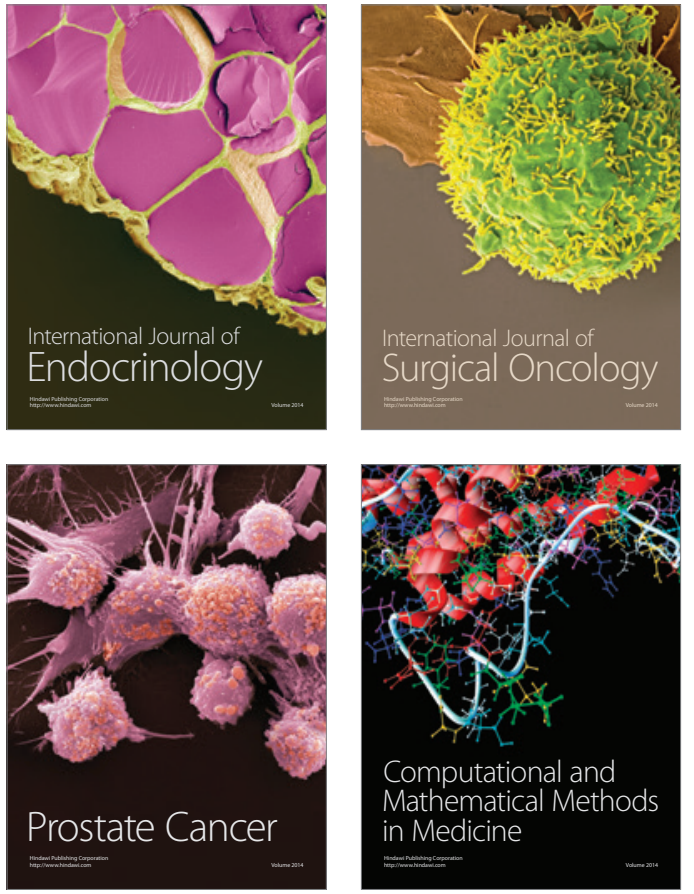
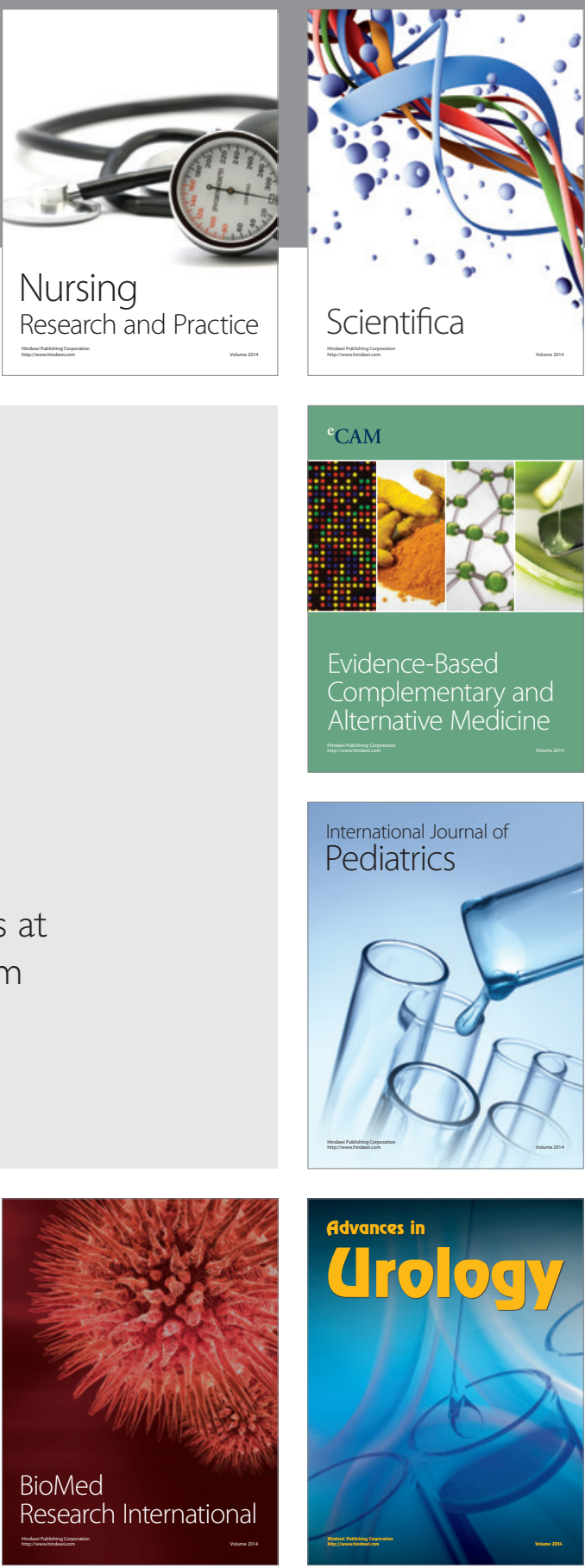

Nursing

Research and Practice

Scientifica

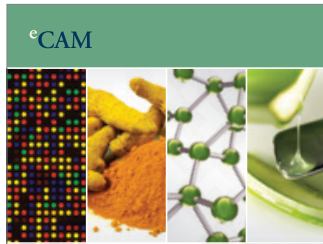

Evidence-Based

Complementary and Alternative Medicine
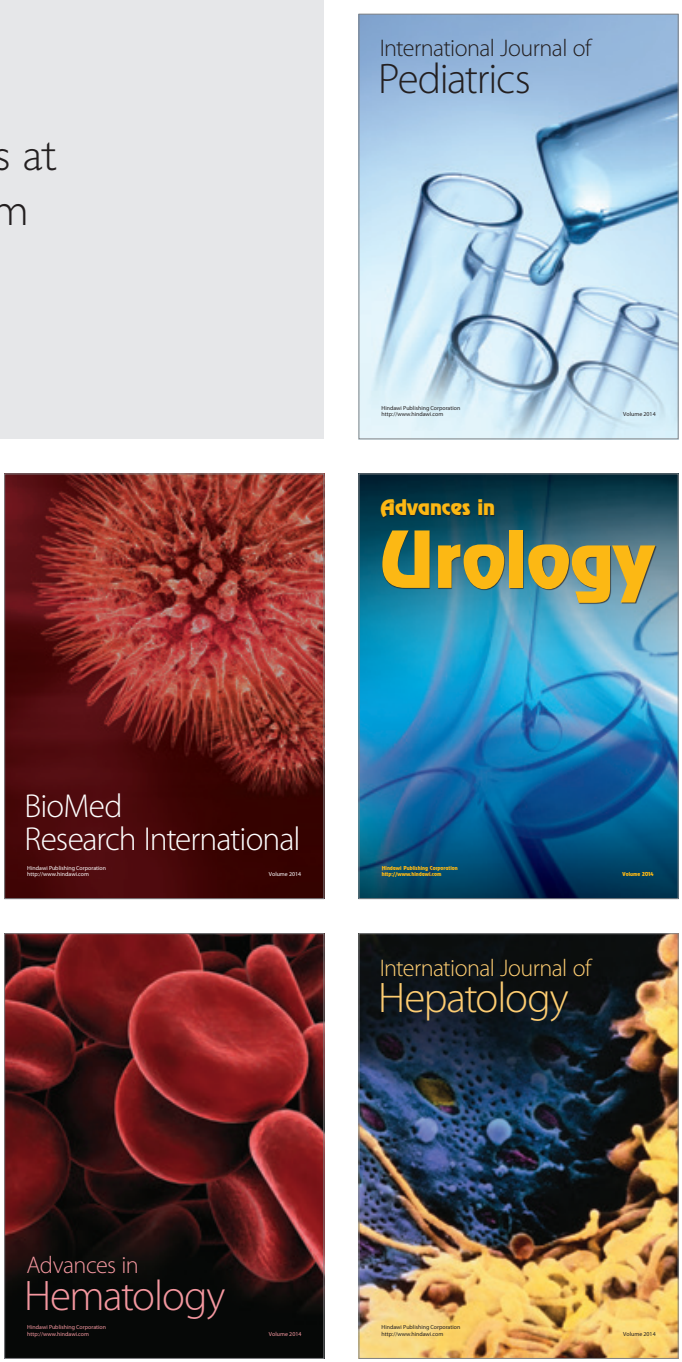